\title{
Evaluación de la calidad de vida en mujeres de 40 a 59 años mediante la escala MRS (Menopause Rating Scale)
}

\author{
Mónica del Prado $A^{a}$, Andrea Fuenzalidaa, Daniela Jara ${ }^{a}$ \\ Rodrigo Figueroa Ja, Daniel Flores ${ }^{1}$, Juan E Blumel $\mathbf{M}^{2}$. \\ Assessment of quality of life using \\ the Menopause Rating Scale \\ in women aged 40 to 59 years
}

Background: Climacteric symptoms have a direct relationship with biological and sociocultural factors and significantly impair the quality of life of women. Aim: To assess quality of life and factors affecting it in women aged 40 to 59 years. Material and methods: The Menopause Rating Scale (MRS) was applied to 370 healthy women aged 49 \pm 6 years, that accompanied patients to public hospitals in Santiago. Results: Forty four percent of women were postmenopausal and $6 \%$ used hormone replacement therapy. Half of the group had less than 12 years of formal education and $67 \%$ had a couple. The mean number of children was $2.8 \pm 1.5$. Total MRS score was $16.2 \pm 8.5$. The higher score was given by the psychological domain $(7.7 \pm 4.4)$, followed by the somatic domain $(5.8 \pm 3.5)$. The urogenital domain had the lowest score $(2.7 \pm 2.9)$. Eighty percent of women had moderate to severe climacteric symptoms. A logistic regression analysis showed that the postmenopausal condition was the factor that caused the greatest derangement in quality of life, followed by her parity. Formal education had the lowest impact. Conclusions: In this sample of women, menopause significantly deteriorated quality of life and sociocultural factors such as the parity also had an impact (Rev Méd Chile 2008; 136: 1511-7).

(Key w ords: Menopause; Quality of life; Questionnaires)

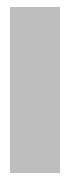

\footnotetext{
Recibido el 2 de mayo, 2008. Aceptado el 25 de agosto, 2008.

${ }^{1}$ Departamento de Gineco-obstetricia, Escuela de Medicina, Universidad Diego Portales, Santiago, Chile. ${ }^{2}$ Departamento Medicina Sur, Facultad de Medicina, Universidad de Chile, Hospital Barros Luco-Trudeau, Santiago, Chile.

${ }^{a}$ Interno de Medicina, Escuela de Medicina, Universidad Diego Portales, Santiago, Chile.
}

$\mathrm{L}$ os cambios biológicos que se producen durante la transición menopáusica ${ }^{1}$ no sólo provocan irregularidades del ciclo menstrual sino tam-

$\overline{\text { Correspondencia a: Mónica del Prado A. Américo Vespucio }}$ Norte 280, Depto. 402. Las Condes. Santiago, Chile. E mail: monica.delprado@gmail.com bién otras manifestaciones, incluyendo síntomas vasomotores, psicológicos y genitourinarios, que afectan profundamente la calidad de vida ${ }^{2}$. En la literatura se relaciona la intensidad de estos síntomas con la presencia de condiciones biopsico-sociales, que pueden modular la calidad de $v_{\text {vida }}^{3,4}$. Entre estos factores, destacan la edad y el nivel sociocultural, características que han sido 
consideradas factores de riesgo independientes para la severidad de la sintomatología climatérica ${ }^{5}$.

Diversas herramientas se han diseñado para la medición y reconocimiento del impacto de estos síntomas en la calidad de vida ${ }^{6}$. El Menopause Rating Scale (MRS) es un instrumento validado de medición de calidad de vida, desarrollado inicialmente a principios de la década 1990-99 para medir la severidad de los síntomas relacionados con la menopausia. Estudios realizados en 9 países y 4 continentes han usado la escala MRS para evaluar la calidad de vida en mujeres durante el climaterio ${ }^{7}$. En Chile, la escala MRS fue validada por Aedo ${ }^{8}$. En Internet es posible acceder al instrumento traducido a 25 idiomas en http://www.menopause-ratingscale.info/; (accedido el 2 de abril de 2008).

El objetivo del presente estudio es evaluar la calidad de vida, mediante la escala MRS, en mujeres entre 40 y 59 años de edad y estudiar la influencia de factores bio-psico-sociales en la calidad de vida.

\section{MATERIAL Y MÉTODO}

Se realizó un estudio descriptivo de corte transversal, llevado a cabo entre agosto y noviembre del año 2007. Se enrolaron mujeres de 40 a 59 años que acudieron como acompañantes a Centros de Salud (Hospital San José, Hospital Dipreca, Hospital de Asistencia Pública) de Santiago. Para el cálculo del tamaño muestral utilizamos el programa estadístico Epi-Info 6.04, módulo Stat calc; con una frecuencia esperada de síntomas climatéricos de $69 \% 9$, con una población estimada en la ciudad de Santiago de 600.000 mujeres entre 40 y 59 años $^{10}$, un error máximo aceptable de $10 \%$ del estimado y 95\% de confianza, se obtuvo que el tamaño mínimo de la muestra debiera ser 168 personas. El muestreo se realizó de forma no aleatoria, por conveniencia, de modo tal que se enrolaron en nuestro estudio a todas las mujeres que cumplían los criterios de inclusión en la fecha determinada.

Los criterios de inclusión consideraron a mujeres entre 40 y 59 años que tuvieran una salud normal, definida según el National Center for Health Statistics como aquella compatible con las actividades rutinarias de una persona ${ }^{11}$. Se excluyeron las mujeres con déficit mental, que les impidiera comprender el cuestionario.
En relación a las características generales de la población se consultaron: edad, años de estudio, estado civil, sistema de atención de salud, presencia o no de menstruaciones, paridad y uso de terapia hormonal de reemplazo.

El instrumento utilizado fue la Escala de Puntuación Menopáusica (MRS), cuestionario compuesto por once síntomas y dividida en tres dominios: 1. Somático: incluye bochornos, sudoración excesiva, molestias cardiacas, trastornos del sueño, molestias musculares y de las articulaciones (items 1-3 y 11, respectivamente); 2. Psicológico: estado depresivo, irritabilidad, ansiedad, cansancio físico y mental (items 4-7, respectivamente); 3. Urogenital: problemas sexuales, de vejiga y sequedad vaginal (items 8-10, respectivamente). Para cada ítem la mujer otorgó un grado de 0 a 4 (0 =ausente; 1 =leve; 2 =moderado; 3 =severo; 4 =muy severo). Para una persona en particular, el puntaje de un dominio corresponde a la sumatoria de los valores obtenidos de cada ítem de esa subescala. El puntaje MRS total será la suma de los puntajes obtenidos de cada dominio ${ }^{12}$.

Se solicitó consentimiento informado previo a la aplicación de la encuesta.

Estadística. Se utilizó el programa estadístico EpiInfo 6.04 y la versión 2002 para Windows; expresándose los resultados en números, porcentajes y media con desviación estándar. Se utilizaron ANOVA y Chi cuadrado para la comparación de las variables continuas y categóricas, respectivamente. Se desarrolló un modelo de regresión logística en el Epi-info 2000, para la evaluación simultánea de la influencia de diferentes variables sobre la calidad de vida. En este modelo se consideraron como variables dependientes los dominios sintomáticos y el puntaje total, definiéndose como calidad de vida alterada un puntaje sobre la mediana. Como variables independientes se consideraron: edad ( $>48$ años), escolaridad ( $>11$ años de estudio), paridad ( $>2$ hijos) y postmenopausia. Un valor de $\mathrm{p}<0,05$ fue considerado estadísticamente significativo.

\section{Resultados}

Se invitó a participar en este estudio a 412 mujeres, de las cuales 42 (10\%) rechazaron partici- 
par. Por tanto, la encuesta se aplicó a 370 mujeres. La edad promedio del grupo fue de 49,3 \pm 5,8 años, con un rango de 40 a 59 años, siendo homogénea su distribución. Respecto de las características sociales de la población se debe mencionar que 209 (56,5\%) estaban casadas, 73 (19,7\%) separadas, 43 (11,6\%) convivían con su pareja, 20 $(5,4 \%)$ viudas y $25(6,8 \%)$ eran solteras. Referente a la paridad, $356(96,2 \%)$ tenían hijos, variando desde 0 a 12 hijos, con una media de 2,8 $\pm 1,5$. Del grupo estudiado, 187 (50,5\%) tenían igual o más de 12 años de estudio y $293(79,2 \%)$ se atendían por el Fondo Nacional de Salud. En relación al estado menstrual, $206(55,6 \%)$ eran premenopáusicas, mientras que $164(44,3 \%)$ resultaron ser postmenopáusicas. Sólo $23(6,2 \%)$ de las mujeres eran usuarias de terapia de reemplazo hormonal (TRH) y $20(5,4 \%)$ usaban algún tipo de medicina alternativa con el mismo fin.

En la Tabla 1 se presenta el puntaje promedio de cada síntoma, de cada dominio y el puntaje total de la población encuestada con el MRS. Los síntomas que muestran mayor severidad son los del dominio psicológico, alcanzando un promedio de $7,7 \pm 4,4$ puntos, con una media de $1,9 \pm 1,1$ puntos para cada pregunta de este grupo de síntomas. Luego, se encuentra el dominio somático con 5,8 $\pm 3,5$ puntos y un promedio por pregunta de $1,5 \pm 0,9$. El dominio con menos impacto sintomático fue el urogenital con $2,7 \pm 2,9$ puntos y un promedio de $0,9 \pm 1,0$ por pregunta. El puntaje total de los tres dominios fue de 16,2 $\pm 8,5$.

En la Tabla 2 se muestra el porcentaje de mujeres con distintos grados de severidad de los síntomas climatéricos en la población estudiada. Se observa, de acuerdo al puntaje total del MRS, que $41,1 \%$ de las encuestadas presentaban síntomas climatéricos severos y 39,7\% molestias moderadas; globalmente, más de $80 \%$ presentaba sintomatología moderada o severa. Al desglosar según dominios, se nota que $56,8 \%$ de las mujeres presentaban síntomas severos de tipo psicológico, 20,8\%, somático y $35,7 \%$ urogenital.

La Tabla 3 muestra el impacto de algunos factores de riesgo sobre las áreas de calidad de vida de la escala MRS. El riesgo de tener mala calidad de vida por cualquiera de los síntomas aumentaba significativamente con: la menopausia (OR: 2,48; IC 95\%: 1,62-3,79), tres o más hijos (OR: 2,23; IC 95\%: 1,47-3,40) y la edad (OR: 2,01; IC 95\%: 1,33-3,05); mientras que disminuía con la mayor escolaridad (OR: 0,48; IC 95\%: 0,31-0,73). El factor que tenía mayor impacto en la sintomatología psicológica era el número de hijos (OR: 2,16; IC 95\%: 1,42-3,27), en la sintomatología de tipo somática era la menopausia (OR: 3,12; IC 95\%: 2,03-4,79) y en la sintomatología urogenital la mayor paridad (OR: 2,25; IC 95\%: 1,48-3,42).

La Tabla 4 muestra los resultados de un modelo de regresión logística, donde se observa

Tabla 1. Puntaje del M RS en población estudiada

\begin{tabular}{|llcc|}
\hline D ominio & Síntomas & $\begin{array}{c}\text { Puntaje de síntomas } \\
\text { (media } \pm \mathbf{D E} \text { ) }\end{array}$ & $\begin{array}{c}\text { Puntaje de dominios } \\
\text { (media } \pm \text { D E) }\end{array}$ \\
\hline \multirow{2}{*}{ Somático } & Bochornos & $1,3 \pm 1,3$ & \\
& Palpitaciones & $0,8 \pm 1,1$ & $5,8 \pm 3,5$ \\
& Trastornos del sueño & $1,8 \pm 1,5$ & \\
& Molestias osteo-musculares & $2,0 \pm 1,4$ & $7,7 \pm 4,4$ \\
Psicológico & Depresión & $2,0 \pm 1,4$ & \\
& Irritabilidad & $1,9 \pm 1,3$ & $2,7 \pm 2,9$ \\
& Ansiedad & $1,8 \pm 1,4$ & \\
& Cansancio & $2,0 \pm 1,4$ & $16,2 \pm 8,5$ \\
\hline
\end{tabular}


Tabla 2. Porcentaje de mujeres con diferentes niveles de severidad de síntomas según M RS

\begin{tabular}{|c|c|c|c|c|c|c|c|c|}
\hline \multirow[t]{2}{*}{$\begin{array}{l}\text { Severidad } \\
\text { de síntomas }\end{array}$} & \multicolumn{2}{|c|}{$\begin{array}{l}\text { Dominio } \\
\text { Somático }\end{array}$} & \multicolumn{2}{|c|}{$\begin{array}{l}\text { Dominio } \\
\text { Psicológico }\end{array}$} & \multicolumn{2}{|c|}{$\begin{array}{l}\text { Dominio } \\
\text { Urogenital }\end{array}$} & \multicolumn{2}{|c|}{$\begin{array}{l}\text { Puntaje } \\
\text { Total }\end{array}$} \\
\hline & Puntos & $\% \pm I C 95 \%$ & Puntos & $\% \pm$ IC $95 \%$ & Puntos & $\% \pm$ IC $95 \%$ & Puntos & $\% \pm$ IC $95 \%$ \\
\hline Ausentes & $0-2$ & $17,0 \pm 4,0$ & $0-1$ & $5,9 \pm 2,6$ & 0 & $35,1 \pm 5,0$ & $0-4$ & $6,8 \pm 2,7$ \\
\hline Leve & $3-4$ & $18,9 \pm 4,2$ & $2-3$ & $14,9 \pm 3,8$ & 1 & $11,1 \pm 3,3$ & $5-8$ & $12,4 \pm 3,5$ \\
\hline Moderado & $5-8$ & $43,2 \pm 5,2$ & $4-6$ & $22,4 \pm 4,4$ & $2-3$ & $18,1 \pm 4,1$ & $9-16$ & $39,7 \pm 5,1$ \\
\hline Severo & $\geq 9$ & $20,8 \pm 4,3$ & $\geq 7$ & $56,8 \pm 5,2$ & $\geq 4$ & $35,7 \pm 5,0$ & $\geq 17$ & $41,1 \pm 5,1$ \\
\hline
\end{tabular}

Tabla 3. Factores de riesgo de deterioro de la calidad de vida (análisis univariado)

\begin{tabular}{|lcccc|}
\hline Factor de riesgo & $\begin{array}{c}\text { Dominio } \\
\text { Somático } \\
\text { OR (IC: 95\%) }\end{array}$ & $\begin{array}{c}\text { Dominio } \\
\text { Psicológico } \\
\text { OR (IC: 95\%) }\end{array}$ & $\begin{array}{c}\text { Dominio } \\
\text { Urogenital } \\
\text { OR (IC: 95\%) }\end{array}$ & $\begin{array}{c}\text { Total } \\
\text { OR (IC: 95\%) }\end{array}$ \\
\hline Postmenopausia & $3,12(2,03-4,79)$ & $1,91(1,26-2,90)$ & $1,69(1,11-2,56)$ & $2,48(1,62-3,79)$ \\
Paridad $\geq 3$ hijos & $1,68(1,11-2,54)$ & $2,16(1,42-3,27)$ & $2,25(1,48-3,42)$ & $2,23(1,47-3,40)$ \\
Edad $\geq 49$ años & $2,84(1,86-4,33)$ & $1,48(0,98-2,23)$ & $1,46(0,97-2,21)$ & $2,01(1,33-3,05)$ \\
Uso de TH & $1,52(0,64-3,62)$ & $1,54(0,60-3,66)$ & $1,66(0,68-4,01)$ & $1,96(0,78-4,87)$ \\
Estudios $>12$ años & $0,55(0,36-0,84)$ & $0,73(0,49-1,11)$ & $0,75(0,49-1,13)$ & $0,48(0,31-0,73)$ \\
\hline
\end{tabular}

OR: Odds ratio, IC: intervalos de confianza.

Tabla 4. Factores de riesgo de deterioro de la calidad de vida (regresión logística)

\begin{tabular}{|lcccc|}
\hline Factor de riesgo & $\begin{array}{c}\text { D ominio } \\
\text { Somático } \\
\text { OR (IC: 95\%) }\end{array}$ & $\begin{array}{c}\text { D ominio } \\
\text { Psicológico } \\
\text { OR (IC: 95\%) }\end{array}$ & $\begin{array}{c}\text { D ominio } \\
\text { Urogenital } \\
\text { OR (IC: 95\%) }\end{array}$ & OR (IC: 95\%) \\
\hline Postmenopausia & $2,09(1,19-3,66)$ & $1,74(1,13-2,66)$ & $1,52(0,99-2,32)$ & $2,18(1,41-3,38)$ \\
Paridad $\geq 3$ hijos & $1,28(0,82-1,99)$ & $1,99(1,31-3,06)$ & $2,12(1,38-3,24)$ & $1,85(1,19-2,86)$ \\
Edad $\geq 49$ años & $1,73(0,99-3,02)$ & $0,84(0,47-1,47)$ & $0,94(0,54-1,66)$ & $1,10(0,62-1,95)$ \\
Estudios $>12$ años & $0,61(0,39-0,93)$ & $0,88(0,57-1,35)$ & $0,89(0,58-1,37)$ & $0,57(0,37-0,87)$ \\
\hline
\end{tabular}

OR: Odds ratio, IC: intervalos de confianza.

que la postmenopausia es el factor que más deteriora la calidad de vida en forma global. Las mujeres postmenopáusicas tenían mayor riesgo de tener un puntaje total sobre la mediana en la escala MRS (OR: 2,18; IC 95\%: 1,41-3,38), encontrándose en segundo lugar, las mujeres con mayor paridad (OR: 1,85; IC 95\%: 1,19-2,86). La edad no se presentaba como un factor significativo de deterioro y las mujeres con más escolaridad tenían menos riesgo de alteración de su calidad de vida (OR: 0,57; IC 95\%: 0,37-0,87). Al desglosarlo por dominios, el factor que tenía mayor impacto en la sintomatología de tipo somática era la menopausia (OR: 2,09; IC 95\%: 1,19-3,66), en la sintomato- 
logía psicológica la mayor paridad (OR: 1,99; IC 95\%: 1,31-3,06), al igual que para la sintomatología urogenital (OR: 2,12; IC 95\%: 1,38-3,24).

\section{DisCUSIÓN}

La mayoría de las mujeres considera que la menopausia tiene efectos negativos físicos y psíquicos en la salud ${ }^{13}$. Sin embargo, en la práctica clínica la evaluación sistemática de estos efectos es muy superficial. Un avance importante en este sentido ha sido el desarrollo de la escala MRS, un instrumento que evalúa el impacto de varios grupos de síntomas en la calidad de vida de la mujer climatérica. En la actualidad en Medline figuran 37 estudios médicos con este instrumento ${ }^{14}$ y se encuentra validado a 25 idiomas $^{15}$.

Al comparar nuestros resultados con los publicados en la página web de la escala MRS se observa que mientras que en Europa, Norteamérica, Latinoamérica y Asia el promedio de puntaje de la escala es de alrededor de 9 puntos, nosotros hemos encontrado $16^{16}$. Esta diferencia se da especialmente por una puntuación 4 veces mayor en el promedio de los síntomas psicológicos de nuestras mujeres y de 2 en los síntomas somáticos. Interesantemente, mientras en Asia y Norteamérica-Europa el promedio de puntaje de los síntomas psicológicos es 2,9 y 3,4, respectivamente, en Latinoamérica es más alto, 4,9 puntos. Nuestros puntajes son superiores a los publicados en la página web del MRS, pero son prácticamente idénticos a los descritos por Aedo en la validación del instrumento en Chile ${ }^{8}$. En relación a éste, aunque el objetivo primordial de los trabajos es diferente, sus métodos son similares, lo que se condice con que nuestros resultados sean casi iguales; mientras este autor encontró un puntaje promedio de 16,1 \pm 9,9 en mujeres controles, nosotros encontramos 16,2 $\pm 8,5$. Creemos que esta "coincidencia" valida nuestro trabajo.

El mayor puntaje de sintomatología climatérica descrito en este estudio, traduce una mayor severidad sintomática e impacto del climaterio en la calidad de vida de estas mujeres. Esto se refleja en las cifras de prevalencia de síntomas severos, de acuerdo a la definición de MRS. Mientras en Europa, Norteamérica, Latinoamérica y Asia, el porcentaje de mujeres con síntomas severos es de
$24,3 \%, 22,5 \%, 22,7 \%$ y $9,5 \%$, respectivamente, nosotros encontramos que $41,1 \%$ de nuestras encuestadas tenían síntomas severos ${ }^{9}$. Este alto porcentaje de mujeres con sintomatología importante tiene implicancias epidemiológicas y nos debiera obligar a plantear a nuestras autoridades de salud la necesidad de desarrollar programas específicos para el climaterio.

En este estudio, el análisis univariado nos sugirió la existencia de algunos factores de riesgo de tener mala calidad de vida, como: menopausia, mayor edad, mayor paridad y baja escolaridad; mientras que el uso de terapia hormonal se presentó como un factor protector. El modelo de regresión logística confirmó que la postmenopausia y la paridad aumentaban la posibilidad de tener más sintomatología climatérica, mientras que la mayor escolaridad implicaba menor riesgo de tener deterioro de la calidad de vida. Nosotros, con un instrumento distinto, ya habíamos previamente mostrado que la menopausia era el principal factor de riesgo de tener síntomas que afecten la calidad de vida ${ }^{17}$. Sin embargo, ese estudio no tenía un puntaje total como tiene el MRS y señalaba que el cese de la función ovárica aumentaba 12 veces el riesgo de tener síntomas vasomotores, cuatro veces de síntomas psicosociales, cuatro de síntomas físicos y 5 de disfunciones sexuales. Nuestros resultados actuales son bastante menores, pero apuntan en el mismo sentido, la menopausia deteriora la calidad de vida. Un segundo factor, que vuelve a repetirse como factor de riesgo de deterioro de calidad de vida en el climaterio en nuestros estudios, es la paridad. En el trabajo ya citado previamente, la paridad mayor a dos hijos duplicaba el riesgo de tener síntomas vasomotores o psicosociales que afectaran la calidad de vida. Esta observación coincide con los resultados de un estudio de Freeman quien señala que la mayor paridad se asocia a mayor riesgo de presentar bochornos ${ }^{18}$. Igualmente, el riesgo de disfunción sexual, se incrementa significativamente con la mayor paridad $^{19}$. En relación a la asociación de menor educación con mayor sintomatología climatérica y mala calidad de vida, esta vinculación aparece en distintas culturas. En Turquía un estudio señala al bajo de nivel de educación como uno de los principales factores de riesgo de mala calidad de vida en mujeres climatéricas ${ }^{20}$. Igualmente, Che- 
draui en Ecuador observa que el bajo nivel de escolaridad es un factor importante de riesgo de tener mala calidad de vida en el período climatéri$\mathrm{CO}^{21}$.

Una de las variables que en el modelo de regresión logística no apareció fue el antecedente del uso de terapia hormonal. Diversos estudios randomizados controlados han demostrado que la terapia hormonal es altamente efectiva en aliviar los síntomas menopáusicos y mejorar la calidad de vida ${ }^{22-24}$. En nuestro estudio el grupo de usuarias de terapia hormonal era muy pequeño por lo cual no tuvo el poder estadístico para evaluar el impacto de este tipo de terapia en la calidad de vida, lo que explicaría que no se haya encontrado una asociación significativa entre el uso de esta terapia y la intensidad de los síntomas menopáusicos, constituyendo ésta una limitación del actual trabajo. Otra limitación de nuestro estudio es que la comparación que hacemos de calidad de vida con mujeres de otras poblaciones se basa en que se usó el mismo instrumento y en que se aplicó a mujeres de edad media, pero esta comparación puede estar sesgada por el hecho que nuestra realidad socioeconómica y cultural es diferente a las de otros países. Dado que estos factores inciden claramente en la calidad de vida, se infiere que ellos podrían ser una de las causas que expliquen los diferentes resultados. Reciente-

\section{REFERENCIAS}

1. Burger HG, Dudley EC, Robertson DM, Dennerstein L. Hormonal changes in the menopause transition. Recent Prog Horm Res 2002; 57: 257-75.

2. Buckler H. The menopause transition: endocrine changes and clinical symptoms. J Br Menopause Soc 2005; 11: 61-5.

3. Binfa L, Castelo-Branco C, Blumel Je, Cancelo MJ, Bonilla H, MunOz I ET aL. Influence of psycho-social factors on climacteric symptoms. Maturitas 2004; 48: 425-31.

4. Blumel Je, Castelo-Branco C, Cancelo MJ, Cordova AT, Binfa LE, Bonilla HG et al. Relationship between psychological complaints and vasomotor symptoms during climacteric. Maturitas 2004; 49: 205-10.

5. Chedraui P, Aguirre W, Hidalgo L, Fayad L. Assessing menopausal symptoms among healthy middle aged mente, se presentó en Madrid en el Congreso de la International Menopause Society (Chedraui P, Ojeda E, Blümel JE, Royer M, Espinoza MT, Mostajo D, et al. Quality of life assessment among Latin American middle aged women. 12 $2^{\text {th }}$ World Congress on the Menopause. May 19-23; 2008. Madrid, Spain) un estudio que comparó los datos de esta investigación con los de otros once países de Latinoamérica que aplicaron simultáneamente la misma metodología para estudiar la calidad de vida en mujeres de edad media, observándose que las mujeres chilenas tienen la peor calidad de vida entre los países estudiados. Santiago es una ciudad "hiperurbanizada", condición que se asocia con mayor prevalencia de síntomas depresivos y estrés; ésta podría ser una de las causas de la mala calidad de vida descrita en nuestro estudio ${ }^{25,26}$.

En conclusión, en esta población, de los factores estudiados mediante la escala MRS que afectan la calidad de vida, la menopausia resultó ser el factor estadísticamente más relevante. Factores socioeconómicos como la mayor paridad y la menor escolaridad pueden asociarse a mayor riesgo de deterioro de calidad de vida. Estos resultados debieran instar a los clínicos que atienden a mujeres de edad media a evaluar sistemáticamente la variada sintomatología de la menopausia y su repercusión en la calidad de vida individual. women with the Menopause Rating Scale. Maturitas 2007; 57: 271-8.

6. Zollner YF, Acquadro C, Schaefer M. Literature review of instruments to assess health-related quality of life during and after menopause. Qual Life Res 2005; 14: 309-27.

7. Heinemman K, Ruebig A, Potthof P. The menopause rating scale (MRS): A methodological review. Qual Life Res 2004; 2: 45.

8. Aedo S, Porcie A, Irribarra C. Calidad de vida relacionada con el climaterio en una población chilena de mujeres saludables. Rev Chil Obstet Ginecol 2006; 71: 402-9.

9. Heinemann L. Comparison of "degree of severity" of the MRS and its domains. Menopause rating scale. Center for Epidemiology and Health Research, Berlin. Disponible en: http://www.menopause-ratingscale.info/documents/Ref_Values_CountrGr.pdf. [Consultado el 4 de abril de 2008]. 
10. Censo 2002. Instituto Nacional de Estadística. Chile. Disponible en: http://www.ine.cl/cd2002/index.php. [Consultado el 4 de abril de 2008].

11. Brett KM, Chong Y. Hormone Replacement Therapy: Knowledge and Use in the States United. Hyattsville, Maryland: National Center for Health Statistics, 2001.

12. Kakkar V, Kaur D, Chopra K, Kaur A, Kaur IP. Assessment of the variation in menopausal symptoms with age, education and working/nonworking status in north-Indian sub population using menopause rating scale (MRS). Maturitas 2007; 57: 306-14.

13. Blumel Je, tacla X, Brandt A, Gramegna G, Estartus A. Knowledge and beliefs of the effect of menopause and estrogenic therapy on health. Study in women attending the Barros Luco-Trudeau Hospital. Rev Chil Obstet Ginecol 1994; 59: 10-6.

14. Pubmed. NCBI. Disponible en: http:// www.ncbi.nlm.nih.gov/sites/entrez [Consultado el 7 de abril de 2008].

15. Heinemann L. About the MRS. Menopause rating scale. Center for Epidemiology and Health Research, Berlin. Disponible en: http://www.menopause-rating-scale.info/. [Consultado el 11 de Abril 2008].

16. Heinemann L. Population reference values. Menopause rating scale. Center for Epidemiology and Health Research, Berlin. Disponible en: http:// www.menopause-rating-scale.info/documents/ Int_MeanSD.pdf. [Consultado el 11 de abril de 2008].

17. Blumel Je, Castelo-Branco C, Binfa L, Gramegna G, Tacla X, Aracena B et al. Quality of life after the menopause: a population study. Maturitas 2000; 34 : $17-23$.

18. Freeman EW, Sammel MD, Grisso JA, Battistini M, Garcia-Espagna B, Hollander L. Hot flashes in the late reproductive years: risk factors for Africa American and Caucasian women. J Womens Health Gend Based Med 2001; 10: 67-76.
19. Botros SM, Abramov Y, Miller JJ, Sand PK, Gandhi S, Nickolov A, Goldberg RP. Effect of parity on sexual function: an identical twin study. Obstet Gynecol 2006; 107: 765-70.

20. KaraÇAm Z, SeKer SE. Factors associated with menopausal symptoms and their relationship with the quality of life among Turkish women. Maturitas 2007; 58: 75-82.

21. Chedraui P, Aguirre W, Hidalgo L, Fayad L. Assessing menopausal symptoms among healthy middle aged women with the Menopause Rating Scale. Maturitas 2007; 20; 57: 271-8.

22. Gelfand MM, Moreau M, Ayotte NJ, Hilditch JR, Wong BA, LAu CY. Clinical assessment and quality of life of postmenopausal women treated with a new intermittent progestogen combination hormone replacement therapy: a placebo-controlled study. Menopause 2003; 10: 29-36.

23. Hilditch JR, Lewis J, Ross AH, Peter A, Van Maris B, Franssen E ET AL. A comparison of the effects of oral conjugated equine estrogen and transdermal estradiol-17 beta combined with an oral progestin on quality of life in postmenopausal women. Maturitas 1996; 24: 177-84.

24. Barnabei VM, Cochrane BB, Aragaki AK, Nygaard I, Williams RS, Mcgovern PG, Young RL, Wells EC, O'Sullivan MJ, Chen B, Schenken R, Johnson SR; Women's Health Initiative Investigators. Menopausal symptoms and treatment-related effects of estrogen and progestin in the Women's Health Initiative. Obstet Gynecol 2005; 105 (5 Pt1): 1063-73.

25. HaRpham T. Urbanization and mental health in developing countries: a research role for social scientists, public health professionals and social psychiatrists. Soc Sci Med 1994; 39: 233-45.

26. Paykel ES, Abbott R, Jenkins R, Brugha TS, Meltzer H. Urban-rural mental health differences in great Britain: findings from the national morbidity survey. Psychol Med 2000; 30: 269-80. 